\title{
An Anisotropic Subgrid-Scale Parameterization for Large-Eddy Simulations of Stratified Turbulence
}

\author{
SINA KHANI \\ Applied Physics Laboratory, and School of Oceanography, University of Washington, Seattle, Washington \\ Michael L. WAITE \\ Department of Applied Mathematics, University of Waterloo, Waterloo, Ontario, Canada
}

(Manuscript received 20 October 2019, in final form 6 August 2020)

\begin{abstract}
Subgrid-scale (SGS) parameterizations in atmosphere and ocean models are often defined independently in the horizontal and vertical directions because the grid spacing is not the same in these directions (anisotropic grids). In this paper, we introduce a new anisotropic SGS model in large-eddy simulations (LES) of stratified turbulence based on horizontal filtering of the equations of motion. Unlike the common horizontal SGS parameterizations in atmosphere and ocean models, the vertical derivatives of the horizontal SGS fluxes are included in our anisotropic SGS scheme, and therefore the horizontal and vertical SGS dissipation mechanisms are not disconnected in the newly developed model. Our model is tested with two vertical grid spacings and various horizontal resolutions, where the horizontal grid spacing is comparatively larger than that in the vertical. Our anisotropic LES model can successfully reproduce the results of direct numerical simulations, while the computational cost is significantly reduced in the LES. We suggest the new anisotropic SGS model as an alternative to current SGS parameterizations in atmosphere and ocean models, in which the schemes for horizontal and vertical scales are often decoupled. The new SGS scheme may improve the dissipative performance of atmosphere and ocean models without adding any backscatter or other energizing terms at small horizontal scales.
\end{abstract}

KEYWORDS: Filtering techniques; Large eddy simulations; Model evaluation/performance; Numerical analysis/modeling; Parameterization; Subgrid-scale processes

\section{Introduction}

Large-eddy simulation (LES) is a useful numerical approach for simulations of geophysical turbulence at the small-scale end of the atmospheric mesoscale, oceanic submesoscale, and smaller scales, where there is forward kinetic energy transfer from large to small horizontal scales. These scale ranges are broadly characterized by strong stratification and weak rotation (i.e., stratified turbulence) (e.g., Riley and Lindborg 2008). It has been shown that fundamental characteristics of stratified turbulence that are seen in direct numerical simulation (DNS), such as a $-5 / 3$ spectral slope in the horizontal wavenumber energy spectra (Waite and Bartello 2004; Lindborg 2006; Brethouwer et al. 2007), layered structures with KelvinHelmholtz (KH) instabilities (Bartello and Tobias 2013; Khani and Waite 2016), nonlocal horizontal energy transfer from large scales to the buoyancy scale (Waite 2011; Khani and Waite 2013), and small or negative local Richardson number associated with overturning (Waite and Bartello 2004; Bartello and Tobias 2013), can be captured by LES if the buoyancy scale $L_{b}=2 \pi u_{\mathrm{rms}} / N$ is sufficiently well resolved (i.e., $\Delta<L_{b}$; see Khani and Waite 2014, 2015). Here, $u_{\mathrm{rms}}, N$, and $\Delta$ are the rootmean-square (rms) velocity, buoyancy frequency, and grid spacing, respectively. These and other LES (e.g., Siegel and Domaradzki 1994; Kang et al. 2003; Paoli et al. 2014) used isotropic grid spacing in the horizontal and vertical directions

Corresponding author: Sina Khani, skhani@uw.edu (i.e., $\Delta_{h}=\Delta_{z}$ ). However, coarser and anisotropic grid spacings are usually employed in atmosphere and ocean simulations, which require a different subgrid approach.

In large-scale atmosphere and ocean models, it is not possible to explicitly resolve the buoyancy scale $L_{b}$ in the horizontal direction, due to limits on computation. For example, the horizontal grid spacing $\Delta_{h}$ in global weather prediction models is often around $10 \mathrm{~km}$ or larger, while $L_{b}$ is on the order of $1 \mathrm{~km}$ in the atmosphere (see e.g., Augier and Lindborg 2013; Brune and Becker 2013; Schaefer-Rolffs and Becker 2018). Therefore, it is not computationally feasible to use isotropic grid spacing to resolve $L_{b}$, and therefore different grid spacings in the horizontal and vertical directions (i.e., anisotropic grids) are usually employed. Atmosphere and ocean models typically use finer grid spacing in the vertical direction than in the horizontal (i.e., $\Delta_{z}<\Delta_{h}$ ), and the question of sufficient vertical resolution for capturing the $-5 / 3$ power law in the horizontal wavenumber energy spectrum has been an active area of discussion (see e.g., Brune and Becker 2013; Augier and Lindborg 2013; Waite 2016; Schaefer-Rolffs and Becker 2018; Skamarock et al. 2019). Generally, most atmosphere and ocean models use different dissipation schemes in the horizontal and vertical directions because of the large difference in horizontal and vertical grid spacings. These dissipation schemes are typically independent of one another: for example, models may use the horizontal Smagorinsky subgrid-scale (SGS) model for horizontal mixing and a vertical stability-dependent eddy viscosity, possibly as part of the boundary layer scheme, 
for vertical mixing [see e.g., Griffies and Hallberg 2000; Skamarock et al. 2008, for the Weather Research and Forecasting (WRF) Model and the Modular Ocean Model (MOM), respectively].

In this paper, we use homogeneous stratified turbulence as an idealized problem in which to investigate the consequences of using decoupled horizontal and vertical SGS dissipation schemes in the limit of high vertical resolution. We develop an anisotropic scheme for LES of stratified turbulence based on a scale analysis of the SGS momentum and potential temperature fluxes in stratified turbulence. Initially, we set $\Delta_{z}$ very small (as in DNS) in our LES runs to evaluate the dependence of our new anisotropic scheme on the horizontal grid spacing $\Delta_{h}$ by comparison with a more typical SGS scheme, in which the horizontal and vertical dissipations are treated separately. Next, we study the effects of vertical resolutions in our new scheme. The rest of this paper is composed as follows: the governing equations and mathematical formulations are given in section 2 . Section 3 presents the methodology and numerical setup. Results are shown and discussed in section 4 , followed by conclusions in section 5 .

\section{Governing equations}

The governing equations of motion under the Boussinesq approximation with uniform stratification can be written in the following nondimensional form (as in e.g., Khani and Waite 2013):

$$
\begin{aligned}
\frac{\partial \mathbf{u}}{\partial t}+\mathbf{u} \cdot \nabla \mathbf{u} & =-\nabla p+\frac{1}{\operatorname{Fr}_{\ell}^{2}} \theta \mathbf{e}_{z}+\frac{1}{\operatorname{Re}_{\ell}} \nabla^{2} \mathbf{u}, \\
\nabla \cdot \mathbf{u} & =0, \\
\frac{\partial \theta}{\partial t}+\mathbf{u} \cdot \nabla \theta+w & =\frac{1}{\operatorname{Re}_{\ell} \operatorname{Pr}} \nabla^{2} \theta,
\end{aligned}
$$

where $\mathbf{u}=(u, v, w)$ is the velocity vector; $\theta$ and $p$ are the potential temperature and pressure perturbations, respectively; and $\operatorname{Re}_{\ell}=\mathscr{Q} \ell / \nu, \mathrm{Fr}_{\ell}=\mathscr{Q} /(\ell N)$, and $\operatorname{Pr}=\nu / \kappa$ are the Reynolds, Froude, and Prandtl numbers, respectively. Here, the velocity and length scales are taken to be unity (i.e., $\mathscr{U} b \equiv 1$ and $\ell \equiv 1$ ), and $\nu$ and $\kappa$ are the molecular viscosity and diffusivity, respectively. In LES, the flow variables are filtered using a filtering operator $G$. For example, the filtered velocity field $\overline{\mathbf{u}}$ is defined as follows (e.g., Pope 2000):

$$
\overline{\mathbf{u}}(\mathbf{x}, t)=\int_{D} \mathbf{u}(\mathbf{x}+\mathbf{r}, t) G(\mathbf{r}) d \mathbf{r},
$$

where $\mathbf{x}=(x, y, z)$ are the Cartesian coordinates and $D$ is the spatial domain. Applying the filtering operator $G$ to the equations of motions [Eqs. (1)-(3)] is straightforward except for the nonlinear terms, which lead to the subgrid-scale (SGS) momentum stress:

$$
\tau_{i j}=\overline{u_{i} u_{j}}-\bar{u}_{i} \bar{u}_{j}
$$

and SGS potential temperature flux:

$$
h_{j}=\overline{\theta u_{j}}-\bar{\theta} \bar{u}_{j}
$$

which are not known in terms of the filtered variables and must be parameterized in LES. In summary, the filtered
Navier-Stokes equations under the Boussinesq approximation can be written as

$$
\begin{aligned}
\frac{\partial \bar{u}_{i}}{\partial t}+\frac{\partial}{\partial x_{j}}\left(\bar{u}_{i} \bar{u}_{j}\right) & =-\frac{\partial \bar{p}}{\partial x_{i}}+\frac{1}{\operatorname{Fr}_{\ell}^{2}} \bar{\theta} \mathbf{e}_{z}+\frac{1}{\operatorname{Re}_{\ell}} \frac{\partial^{2} \bar{u}_{i}}{\partial x_{j} \partial x_{j}}-\frac{\partial \tau_{i j}}{\partial x_{j}}, \\
\frac{\partial \bar{u}_{i}}{\partial x_{i}} & =0 \\
\frac{\partial \bar{\theta}}{\partial t}+\frac{\partial}{\partial x_{j}}\left(\bar{\theta} \bar{u}_{j}\right)+\bar{w} & =\frac{1}{\operatorname{Re}_{\ell} \operatorname{Pr}} \frac{\partial \bar{\theta}}{\partial x_{j} \partial x_{j}}-\frac{\partial h_{j}}{\partial x_{j}} .
\end{aligned}
$$

Before introducing SGS models for the momentum and potential temperature fluxes, it is useful to investigate the SGS momentum stress $\tau_{i j}$ using Taylor series and the definition of the filtering operator. A similar procedure is also done for the potential temperature SGS flux $h_{j}$.

We can expand the velocity field $\mathbf{u}(\mathbf{x}+\mathbf{r})$ using a Taylor series at a given point $\mathbf{x}$ in $\mathbf{r}$, which is on the order of the filter width $\Delta$ (see e.g., Pope 2000; Meneveau and Katz 2000; Khani and Porté-Agel 2017a,b):

$$
\begin{aligned}
u_{i}(\mathbf{x}+\mathbf{r})= & u_{i}(\mathbf{x})+\frac{\partial u_{i}(\mathbf{x})}{\partial x_{k}} r_{k}+\frac{1}{2} \frac{\partial^{2} u_{i}(\mathbf{x})}{\partial x_{k} \partial x_{l}} r_{k} r_{l} \\
& +\frac{1}{6} \frac{\partial^{3} u_{i}(\mathbf{x})}{\partial x_{m} \partial x_{k} \partial x_{l}} r_{m} r_{k} r_{l}+\cdots
\end{aligned}
$$

Using this expansion, we can find the following nonlinear approximation for the SGS stress tensor (see appendix A for details):

$$
\tau_{i j}(\mathbf{x}) \approx \frac{\partial u_{i}(\mathbf{x})}{\partial x_{l}} \frac{\partial u_{j}(\mathbf{x})}{\partial x_{k}} \int_{D} r_{k} r_{l} G(\mathbf{r}) d \mathbf{r} .
$$

The SGS stress $\tau_{i j}$ depends on the filtering function $G$ and the integral over the associated domain. For example, if $G$ is an isotropic Gaussian function with variance $\Delta^{2} / 12$, Eq. (11) yields

$$
\tau_{i j}(\mathbf{x}) \approx \frac{\Delta^{2}}{12} \frac{\partial u_{i}(\mathbf{x})}{\partial x_{k}} \frac{\partial u_{j}(\mathbf{x})}{\partial x_{k}} .
$$

Horizontal SGS mixing parameterizes the effects of small unresolved horizontal scales. As a result, it can be investigated by applying a filter to horizontal scales only [i.e., $\left.G=G\left(r_{x}, r_{y}\right)\right]$. In this case, the dummy indices $l$ and $k$ in Eq. (11) will $\operatorname{span}\{1,2\}$ and Eq. (11) will not include $z$ derivatives. Therefore, using an isotropic horizontal Gaussian filter, Eq. (12) becomes

$\tau_{i j}(\mathbf{x}) \approx \frac{\Delta^{2}}{12}\left[\frac{\partial u_{i}(\mathbf{x})}{\partial x} \frac{\partial u_{j}(\mathbf{x})}{\partial x}+\frac{\partial u_{i}(\mathbf{x})}{\partial y} \frac{\partial u_{j}(\mathbf{x})}{\partial y}\right], \quad i, j=1,2,3$.

The vertical components of the SGS stress $\tau_{i j}$ (i.e., $\tau_{13}, \tau_{23}$, and $\left.\tau_{33}\right)$ are not zero because the horizontal derivatives of vertical motions (i.e., $\partial w / \partial x$ and $\partial w / \partial y)$ are nonzero.

Similarly, for the SGS flux term $h_{j}(\mathbf{x})=\overline{\theta(\mathbf{x}) u_{j}(\mathbf{x})}-\bar{\theta}(\mathbf{x}) \bar{u}_{j}(\mathbf{x})$, we can write (see appendix B for more information):

$$
h_{j}(\mathbf{x}) \approx \frac{\partial \theta(\mathbf{x})}{\partial x_{l}} \frac{\partial u_{j}(\mathbf{x})}{\partial x_{k}} \int_{D} r_{k} r_{l} G(\mathbf{r}) d \mathbf{r} .
$$


Again, assuming a horizontal Gaussian filter function $G$, we obtain

$$
h_{j}(\mathbf{x}) \approx \frac{\Delta^{2}}{12}\left[\frac{\partial \theta(\mathbf{x})}{\partial x} \frac{\partial u_{j}(\mathbf{x})}{\partial x}+\frac{\partial \theta(\mathbf{x})}{\partial y} \frac{\partial u_{j}(\mathbf{x})}{\partial y}\right],
$$

where the vertical component of the SGS potential temperature flux $h_{j}$ is nonzero because $\partial w / \partial x$ and $\partial w / \partial y$ are nonzero [similar to Eq. (13)]. Note that we do not use Eqs. (13) and (15) as a parameterization, but rather as a guide to determine what terms in $\tau_{\mathrm{ij}}$ and $h_{j}$ should be retained and parameterized. Overall, from Eqs. (13) and (15) it is clear that vertical components of SGS fluxes are not zero even when the focus is on only unresolved horizontal scales (i.e., with a purely horizontal filtering operator $G$ ).

We use scale analysis to estimate the size of the various terms in Eqs. (13) and (15) in geophysical simulations. Let $l_{h}$ and $l_{z}$ be the horizontal and vertical scales, respectively, where $l_{z} \ll l_{h}$. In this case, the horizontal and vertical components of the SGS tensor $\tau_{i j}$ can be scaled as (recall that $\tau_{i j}$ is symmetric)

$$
\tau_{i j} \sim \begin{cases}\Delta^{2}\left(\frac{U}{l_{h}}\right)^{2} & \text { for } i, j=1,2 \\ \Delta^{2}\left(\frac{U}{l_{h}}\right)^{2}\left(\frac{l_{z}}{l_{h}}\right) & \text { for } \quad i=1,2 \text { and } j=3 \\ \Delta^{2}\left(\frac{U}{l_{h}}\right)^{2}\left(\frac{l_{z}}{l_{h}}\right)^{2} & \text { for } i, j=3,\end{cases}
$$

where tilde $(\sim)$ denotes order of magnitude, $U$ is the horizontal velocity scale, and we have used the continuity equation to scale the vertical velocity as $l_{z} U / l_{h}$ (as in e.g., Riley and Lelong 2000). Using a similar scale analysis, the SGS stress tensor divergence in Eq. (7) can be scaled as

$$
\frac{\partial \tau_{i j}}{\partial x_{j}} \sim \begin{cases}\frac{\Delta^{2}}{l_{h}}\left(\frac{U}{l_{h}}\right)^{2} & \text { for } \quad i, j=1,2 \\ \frac{\Delta^{2}}{l_{h}}\left(\frac{U}{l_{h}}\right)^{2} & \text { for } \quad i=1,2 \text { and } j=3 \\ \frac{\Delta^{2}}{l_{h}}\left(\frac{U}{l_{h}}\right)^{2}\left(\frac{l_{z}}{l_{h}}\right) & \text { for } \quad i, j=3 .\end{cases}
$$

The horizontal derivatives of horizontal stress $(i, j=1,2)$ and vertical derivatives of SGS stresses with $i=1,2$ and $j=3$ are of the same order of magnitude, and therefore the latter terms are not negligible in comparison with the former when a horizontal filter function is employed. Yet these terms, $\partial \tau_{13} / \partial x_{3}$ and $\partial \tau_{23} / \partial x_{3}$, are not included in purely horizontal mixing schemes (e.g., horizontal Smagorinsky in WRF; see Skamarock et al. 2008). Similarly, we can scale the SGS potential temperature flux divergence $\partial h_{j} / \partial x_{j}$ as

$$
\frac{\partial h_{j}}{\partial x_{j}} \sim\left\{\begin{array}{ll}
\frac{\Delta^{2}}{l_{h}}\left(\frac{\Theta U}{l_{h}}\right)^{2} & \text { for } j=1,2 \\
\frac{\Delta^{2}}{l_{h}}\left(\frac{\Theta U}{l_{h}}\right)^{2} & \text { for } j=3
\end{array},\right.
$$

where $\Theta$ is the potential temperature scale. Again, both horizontal and vertical derivatives of $h_{j}$ are of the same order of magnitude.

The SGS term $\partial \tau_{i j} / \partial x_{j}$ includes the following terms in the $x, y$, and $z$ directions, respectively,

$$
\begin{aligned}
& \left(\frac{\partial \tau_{11}}{\partial x}+\frac{\partial \tau_{12}}{\partial y}+\frac{\partial \tau_{13}}{\partial z}\right), \quad x \text { direction, } \\
& \left(\frac{\partial \tau_{12}}{\partial x}+\frac{\partial \tau_{22}}{\partial y}+\frac{\partial \tau_{23}}{\partial z}\right), \quad y \text { direction, } \\
& \left(\frac{\partial \tau_{13}}{\partial x}+\frac{\partial \tau_{23}}{\partial y}\right), \quad z \text { direction },
\end{aligned}
$$

where only the term $\partial \tau_{33} / \partial z x$ is negligible [see Eq. (17)]. Also, the potential temperature flux $\partial h_{j} / \partial x_{j}$ includes the following terms:

$$
\left(\frac{\partial h_{1}}{\partial x}+\frac{\partial h_{2}}{\partial y}+\frac{\partial h_{3}}{\partial z}\right)
$$

where all terms are important [see Eq. (18)]. In later sections, we will perform LES runs with anisotropic dissipation following Eqs. (19) to (22), and compare the results with DNS, and classic LES where the horizontal and vertical dissipation schemes are not connected (i.e., the vertical components $\tau_{13}$, $\tau_{23}$, and $h_{3}$ are omitted).

\section{Methodology}

We consider a domain with periodic boundary conditions. The horizontal side length is $\mathscr{L}_{h}=2 \pi$, and the vertical side length, depending on the simulation, is $\mathscr{L}_{v}=2 \pi$ or $\pi$. Decaying stratified turbulence is simulated: we have scaled the velocity amplitude to set an initial energy of 0.13 with random phases over a spherical wavevector shell $k_{i}-$ $0.5<k \leq k_{i}+0.5$, where $k=\sqrt{k_{x}^{2}+k_{y}^{2}+k_{z}^{2}}$ is the total wavenumber and $k_{i}=3$ is the initial wavenumber (a similar initial condition is used in Bartello and Tobias 2013). The initial potential temperature fluctuation is zero. The buoyancy frequency is $N=2.1$ or 4.2 , both of which ensure small initial Froude numbers in our simulations (i.e., $\left.\mathrm{Fr}_{l}=1 / N\right)$. Grid sizes vary from $n_{x}=n_{y}=240$ to 960 . For the DNS, $n_{z}=n_{x}$ (for $\mathscr{L}_{v}=2 \pi$ ) or $n_{z}=n_{x} / 2$ (for $\mathscr{B}_{v}=\pi$ ), where $n_{i}$ is the number of grid points in the $i=x$, $y$, and $z$ directions. Spatial derivatives are discretized using the spectral transform method, and the two-thirds rule (Orszag 1971) is employed to eliminate aliasing errors, which leads to an effective grid spacing $\Delta_{h}=3 \mathscr{b}_{h} /\left(2 n_{x}\right)$ and $\Delta_{z}=3 \mathscr{b}_{v} /\left(2 n_{z}\right)$. The third-order Adams-Bashforth scheme is employed for the time stepping of all terms in the transport equations, except the molecular dissipation terms, which are treated with a Crank-Nicolson approach (see e.g., Durran 2010).

A new anisotropic ${ }^{1}$ LES method, in which the vertical derivatives of the SGS stress and flux are retained

\footnotetext{
${ }^{1}$ Here, "anisotropic" refers to an SGS closure based on horizontal filtering only.
} 
TABLE 1. List of numerical simulations with DNS and LES.

\begin{tabular}{|c|c|c|c|c|c|c|c|c|c|c|c|c|}
\hline DNS & $N$ & $n_{x, y}$ & $n_{z}$ & $L_{x, y}$ & $L_{z}$ & $\langle\varepsilon\rangle$ & $\left\langle\varepsilon_{p}\right\rangle$ & $\langle E(t)\rangle$ & $\operatorname{Re}_{b}$ & $\mathrm{Fr}_{h}$ & $k_{b}$ & $k_{o}$ \\
\hline D18N2 & 2.1 & 960 & 960 & $2 \pi$ & $2 \pi$ & $7.42 \times 10^{-3}$ & $4.22 \times 10^{-3}$ & 0.074 & 30.3 & 0.048 & 7.7 & 35.3 \\
\hline $\mathrm{D} 22 \mathrm{~N} 4$ & 4.2 & 960 & 480 & $2 \pi$ & $\pi$ & $4.93 \times 10^{-3}$ & $2.67 \times 10^{-3}$ & 0.076 & 6.2 & 0.015 & 15.2 & 122.6 \\
\hline LES & $N$ & $n_{x, y}$ & $n_{z}$ & $L_{x, y}$ & $L_{z}$ & $\langle\varepsilon\rangle$ & $\left\langle\varepsilon_{p}\right\rangle$ & $\langle E(t)\rangle$ & $k_{c}$ & $\mathrm{Fr}_{h}$ & $k_{b}$ & $k_{o}$ \\
\hline LA18N2a & 2.1 & 480 & 960 & $2 \pi$ & $2 \pi$ & $8.34 \times 10^{-3}$ & $4.29 \times 10^{-3}$ & 0.074 & 158 & 0.054 & 7.7 & 33.3 \\
\hline LA 18 N2b & 2.1 & 240 & 960 & $2 \pi$ & $2 \pi$ & $6.94 \times 10^{-3}$ & $3.77 \times 10^{-3}$ & 0.082 & 78 & 0.040 & 7.4 & 36.5 \\
\hline LA22N4a & 4.2 & 480 & 480 & $2 \pi$ & $\pi$ & $5.75 \times 10^{-3}$ & $3.23 \times 10^{-3}$ & 0.079 & 158 & 0.017 & 15.0 & 113.5 \\
\hline LA22N4b & 4.2 & 240 & 480 & $2 \pi$ & $\pi$ & $5.21 \times 10^{-3}$ & $3.15 \times 10^{-3}$ & 0.082 & 78 & 0.015 & 14.7 & 119.2 \\
\hline LAV18N2b & 2.1 & 240 & 480 & $2 \pi$ & $2 \pi$ & $6.69 \times 10^{-3}$ & $3.75 \times 10^{-3}$ & 0.081 & 78 & 0.036 & 7.1 & 37.2 \\
\hline LAV18N2c & 2.1 & 120 & 480 & $2 \pi$ & $2 \pi$ & $7.10 \times 10^{-3}$ & $3.86 \times 10^{-3}$ & 0.078 & 38 & 0.043 & 7.5 & 36.1 \\
\hline LAV18N2d & 2.1 & 160 & 480 & $2 \pi$ & $2 \pi$ & $6.26 \times 10^{-3}$ & $3.41 \times 10^{-3}$ & 0.083 & 52 & 0.036 & 7.3 & 38.5 \\
\hline
\end{tabular}

as shown in Eqs. (19)-(22), is tested. We employ the dynamic Smagorinsky SGS model because it has the best overall performance in comparison with other SGS parameterizations in LES of stratified turbulence (see Khani and Waite 2014, 2015). The eddy viscosity and diffusivity terms in the anisotropic dynamic Smagorinsky model are given as follows:

$$
\begin{aligned}
& \frac{\partial}{\partial x}\left(2 \bar{S} \bar{s}_{11}\right)+\frac{\partial}{\partial y}\left(2 \bar{S} \bar{s}_{12}\right)+\frac{\partial}{\partial z}\left(2 \bar{S} \bar{s}_{13}\right), \\
& x \text {-direction momentum equation, } \\
& \frac{\partial}{\partial x}\left(2 \bar{S}_{12}\right)+\frac{\partial}{\partial y}\left(2 \bar{S} \bar{s}_{22}\right)+\frac{\partial}{\partial z}\left(2 \bar{S} \bar{s}_{23}\right), \\
& y \text {-direction momentum equation, } \\
& \frac{\partial}{\partial x}\left(2 \bar{S}_{13}\right)+\frac{\partial}{\partial y}\left(2 \bar{S}_{23}\right), \\
& z \text {-direction momentum equation, }
\end{aligned}
$$

and

$$
\begin{gathered}
\frac{\partial}{\partial x}\left(\frac{\bar{S}}{\operatorname{Pr}_{t}} \frac{\partial \bar{\theta}}{\partial x}\right)+\frac{\partial}{\partial y}\left(\frac{\bar{S}}{\operatorname{Pr}_{t}} \frac{\partial \bar{\theta}}{\partial y}\right)+\frac{\partial}{\partial z}\left(\frac{\bar{S}}{\operatorname{Pr}_{t}} \frac{\partial \bar{\theta}}{\partial z}\right) \\
\text { potential temperature equation, }
\end{gathered}
$$

where

$$
\bar{s}_{i j}=(1 / 2)\left(\partial \bar{u}_{i} / \partial x_{j}+\partial \bar{u}_{j} / \partial x_{i}\right)
$$

is the rate-of-strain tensor, $\bar{S}=-c_{s} \Delta_{h}^{2}\left(2 \bar{s}_{i j} \bar{s}_{i j}\right)^{1 / 2}$ is proportional to the total strain rate, $c_{s}$ is the time and space dependent Smagorinsky coefficient, which is calculated using an isotropic explicit coarse filter scale $\tilde{\Delta}=2 \Delta_{h}$ (negative $c_{s}$ values are clipped, see Khani and Waite 2015); the turbulent Prandtl number $\operatorname{Pr}_{t}=1$. Since the anisotropic LES approach is based on horizontal-only filtering, these simulations use high (DNS) resolution in the vertical.

For comparison, classic LES and DNS runs are also performed. In the classic LES runs, the vertical dissipation scale is resolved with high (DNS) vertical resolution, and therefore terms including vertical derivatives in Eqs. (23)-(26) are neglected in the classic horizontal SGS parameterization. The spatial resolution of DNS runs is high in all directions and no SGS model is included. The DNS resolution of the Kolmogorov scale $L_{d}$ is $k_{\max } / k_{d} \gtrsim 0.67$, which is in line with the criterion given by Moin and Mahesh (1998). Here, $k_{\max }$ is the maximum wavenumber and $k_{d}$ is the Kolmogorov wavenumber, calculated over the time interval of maximum kinetic energy dissipation. Our setup with small $\Delta_{z}$ (DNS) is not standard in atmosphere and ocean simulations-indeed, the vertical grids in such models do not ever resolve the Kolmogorov scale-but it is a sensible experiment in which to investigate the parameterization of subgrid horizontal scales. In current SGS models, which we call here classic LES, the limit of $\Delta_{z} \rightarrow 0$ would shut off the requirement for a vertical mixing scheme; here we show that simulations with the classic LES model will fail in such a limit, for example, the kinetic energy spectra are underdissipated (see below), thereby illustrating a problem with the classic approach.

Simulation results are averaged over a time interval around which the kinetic energy dissipation rate $\epsilon$ is maximum. The rms velocity $u_{\mathrm{rms}}=\sqrt{\langle E(t)\rangle}$, where the angle bracket $\langle\cdot\rangle$ denotes time averaging and $E(t)$ is the domainaveraged kinetic energy. The buoyancy scale $L_{b}=2 \pi u_{\mathrm{rms}} / N$, the Ozmidov scale $L_{o}=2 \pi\left(\varepsilon / N^{3}\right)^{1 / 2}$, and the Kolmogorov scale $L_{d}=2 \pi\left(v^{3} / \varepsilon\right)^{1 / 4}$. In our LES, unless there is no eddy viscosity/diffusivity terms (i.e., in cases with high vertical resolution $\Delta_{z}$ ), molecular viscosity and diffusivity are switched off. Table 1 shows a list of averaged variables and parameters for the DNS and anisotropic LES runs. ${ }^{2}$ In the rest of this paper, simulation names start with " $D$ " for DNS, "LA" for anisotropic LES with the same vertical resolution as DNS but coarser horizontal resolution, and "LAV" for anisotropic LES with half the vertical resolution compared with "LA" runs (see Table 1). For LAV runs, the Kolmogorov scale is not resolved in the vertical direction, and there is no extra vertical SGS parameterization (only the anisotropic SGS model is employed). The classic LES simulations, which are labeled with "LC" (see the caption of

\footnotetext{
${ }^{2}$ Simulations in Table 1 are named by their initial Reynolds numbers $\mathrm{Re}_{l}$ and buoyancy frequencies $N\left(=1 / \mathrm{Fr}_{l}\right)$.
} 
(a)

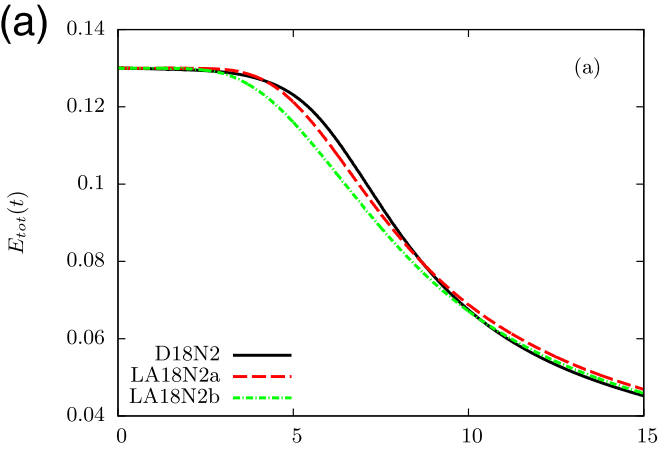

(c)

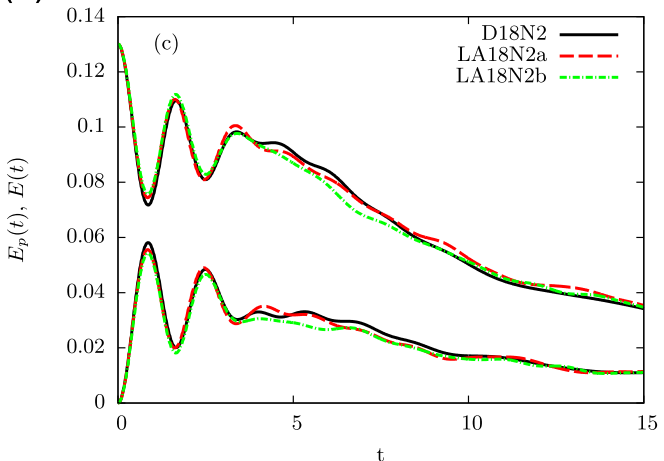

(b)

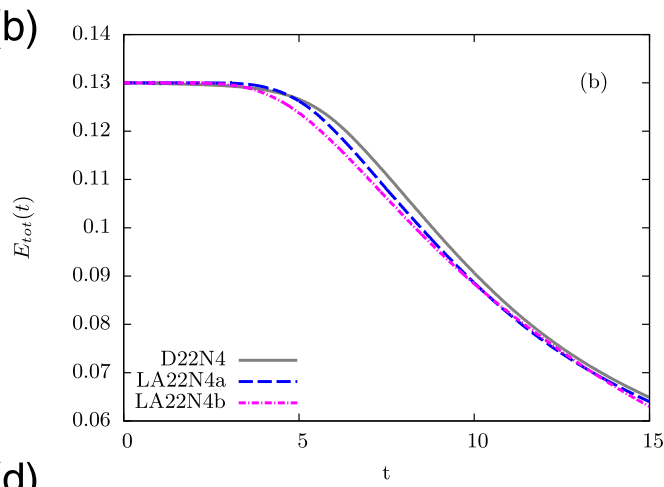

(d)

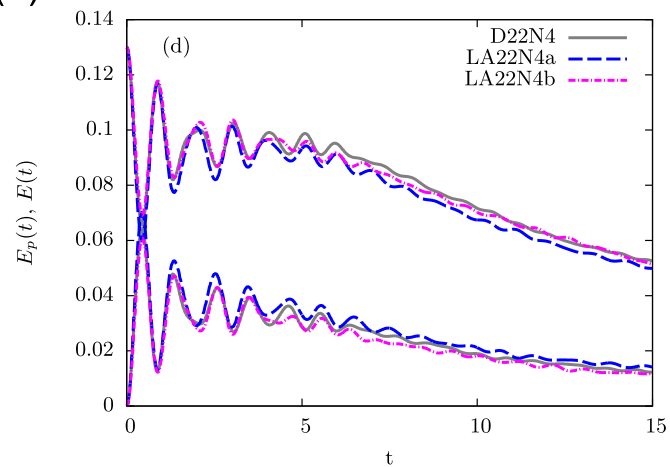

FIG. 1. Time series of (top) total energy and (bottom) the kinetic and potential energy for DNS and anisotropic LES runs with (a),(c) weak and (b),(d) strong stratification. Potential energy curves in (c),(d) are those that start from zero.

Fig. 5), are not reported in Table 1 because these runs are underdissipated (see below).

\section{Results and discussion}

\section{a. Overview of simulations}

Figures 1a-d show the time evolution of the total, kinetic, and potential energy, respectively, for the DNS and anisotropic LES runs. The total energy is almost constant up to approximately $t=5$, and then it decays due to the onset of turbulence (see below). The time series of the kinetic and potential energy (KE and PE) show oscillations, mainly before turbulence decay, due to buoyancy exchanges between KE and PE, since only KE is present at $t=0$ (Figs. $1 \mathrm{c}, \mathrm{d}$ ). The oscillation time scale is related to the frequency of the gravity waves excited by the initial conditions, and therefore becomes smaller in the case with stronger stratification (Figs. 1c,d). The anisotropic LES runs correctly capture the energy oscillations and onset of dissipation from the DNS runs, although the anisotropic LES cases with coarser horizontal resolution (i.e., cases LA18N2b and LA22N4b) slightly underestimate the total energy level after the occurrence of turbulence, which is due to larger eddy dissipation in these coarse LES simulations. Moreover, the onset of turbulence happens earlier in the anisotropic LES cases with the lowest horizontal resolution (see green and magenta dash-dot lines in Figs. 1a,b).

The kinetic energy dissipation rate $\varepsilon$ for cases with the initial Reynolds number $\operatorname{Re}_{l}=18000$, buoyancy frequency $N=2.1$, and $\mathrm{Re}_{l}=22200, N=4.2$ are displayed in Figs. 2a and 2b, respectively. The solid black line in Fig. 2a shows $\varepsilon$ for the DNS run, to which we compare the $\varepsilon$ in the anisotropic LES cases (red dash and green dash-dot lines). A similar comparison is provided in Fig. 2b, in which the solid gray line shows $\varepsilon$ for the DNS case, and the blue dash and magenta dash-dot lines show $\varepsilon$ in the anisotropic LES runs. The kinetic energy dissipation rate has a maximum around $t=7$ and $t=8$ in Figs. $2 \mathrm{a}$ and $2 \mathrm{~b}$, respectively, for the DNS runs. These maxima give the approximate time at which turbulence onset occurs. The onset time for turbulence is relatively well estimated by the anisotropic LES cases with finer horizontal resolutions (i.e., cases LA18N2a and LA22N4a), although the magnitudes of $\varepsilon$ at the maximum times are a little higher in these LES cases in comparison with the DNS. If the horizontal grid spacing decreases further in the anisotropic LES cases (LA18N2b and LA22N4b), turbulence onset occurs earlier while the maximum $\varepsilon$ values would be around or a little smaller than the corresponding DNS runs (solid versus dash-dot line in Fig. 2). Overall, the anisotropic LES runs show larger $\varepsilon$ at early times compared to the DNS cases, but differences between the kinetic energy dissipation rates in the DNS and anisotropic LES cases are smaller after the onset of turbulence (Fig. 2).

Figures 3 and 4 show the $y$ component of vorticity on the $x-z$ plane at $y=0.25$ and $t=15$ for weak and strong stratification cases, respectively (Figs. 3a and 4a for DNS and Figs. 3b,c and $4 b, c$ for anisotropic LES). In the DNS, the vorticity snapshot shows layers, $\mathrm{KH}$ instabilities, and regions of more isotropic 
(a)

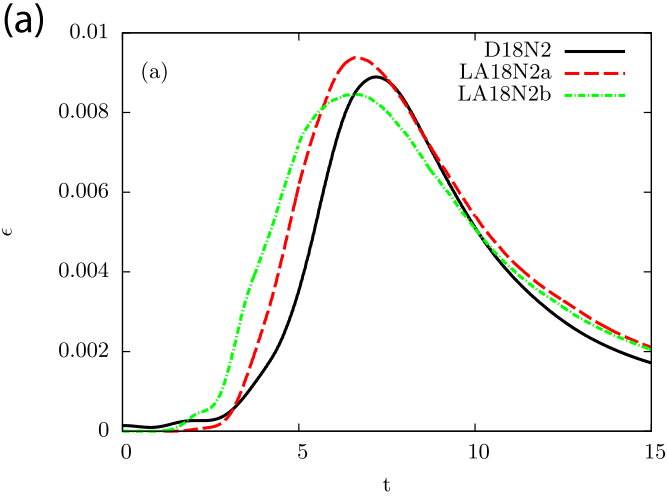

(b)

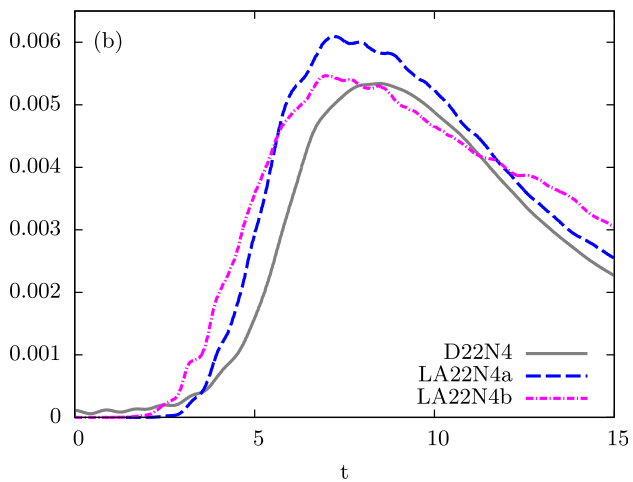

FIG. 2. Time series of the kinetic energy dissipation rate for DNS and anisotropic LES runs with (a) weak and (b) strong stratification.

small-scale turbulence (see e.g., regions around $z=2$ and $x=$ [0-3], or $z \approx 0.8$ and $x=[5-6]$ in Fig. 4a). Layering is more pronounced in the simulation with larger stratification, which has smaller $\mathrm{Re}_{b}$ and is therefore more influenced by dissipation (as shown in Fig. 4a, and also see Brethouwer et al. 2007; Bartello and Tobias 2013; Khani and Waite 2014). For example, Fig. $3 b$ depicts many regions with small-scale isotropic turbulence, while layered structures are more visible in Fig. 4b where stratification is increased. If we further decrease the horizontal resolution in the anisotropic LES, similar largescale structures are generally seen in both weak and strong stratification cases. Interestingly, the anisotropic LES runs with larger grid spacing (i.e., coarser resolution compared to DNS) reproduce similar structures that are seen in the DNS runs with smaller grid spacing. The horizontal layers are much more pronounced in these low-resolution simulations due to significantly larger horizontal dissipation because $\Delta_{h}$ is much larger here, which reduces the transition to small-scale isotropy (Figs. 3c and 4c).

\section{b. Kinetic energy spectra}

The horizontal and vertical wavenumber kinetic energy spectra for DNS, anisotropic LES, and classic LES runs are shown in Fig. 5 (Figs. 5a,b show simulations with $\mathrm{Re}_{l}=$ 18000 and $N=2.1$, and Figs. 5c,d show simulations with $\operatorname{Re}_{l}=22200$ and $N=4.2$ ). The spectra are averaged over a time interval $\Delta t=4$ around the maximum kinetic energy dissipation rate. The high-resolution anisotropic LES (LA18N2a and LA22N4a cases) show almost identical vertical wavenumber kinetic energy spectra to those for DNS (red and blue dashed versus black and gray solid lines in Figs. 5b,d). This trend may not be unexpected since both the DNS and high-resolution anisotropic LES have the same vertical resolution, but the horizontal resolutions are different. The horizontal wavenumber kinetic energy spectra of the anisotropic LES and DNS are also very similar with higher horizontal resolution in the LES (with $\Delta_{h}^{\mathrm{LA}}=2 \Delta_{h}^{D}$; red and blue dashed versus black and gray solid lines in Figs. 5a,c). If we further reduce the horizontal resolution in the anisotropic LES to $4 \Delta_{h}^{D}$, we still can obtain reasonable results at large scales, although both the vertical and horizontal wavenumber kinetic energy spectra are less energetic at smaller scales due to the larger eddy dissipation at small horizontal scales (see green and magenta dash-dotted lines in Fig. 5). Overall, the anisotropic LES model is able to capture the inertial subrange of stratified turbulence similar to the DNS, with significantly less computational cost. Nevertheless, the kinetic energy spectra in the coarse horizontal-resolution cases (LA18N2b and LA22N4b) are more steep at small resolved scales.

The coarser resolution anisotropic LES cases have more dissipation at small vertical scales, as evidenced by the steeper vertical spectra, in comparison with DNS (or highresolution anisotropic LES), despite the fact that they have the same $\Delta_{z}$. This behavior suggests that horizontal resolution can have a significant impact on the resolution of small vertical scales in LES of stratified turbulence, and that the dissipation mechanisms in the horizontal and vertical directions are actually connected.

If the vertical derivatives of the SGS stress and flux are omitted in our LES runs (i.e., classic LES), the impact on the kinetic energy spectra are significant. Indeed, the spectra are underdissipated; there is insufficient small-scale dissipation and, as a result, energy accumulates around the smallest resolved scales in both horizontal and vertical wavenumber spectra (see cyan lines in Fig. 5). The vertical derivatives of SGS fluxes, which are missing in the classic LES runs, therefore play an important role in removing energy from small horizontal and vertical scales. Neglecting these terms can lead to unrealistic results, even with fine (DNS) grid spacings in the vertical. Overall, the results of this section show that the scale analyses in Eqs. (17) and (18), which lead to the anisotropic LES parameterizations that are shown by Eqs. (19)-(22), are confirmed using numerical simulations.

If we further reduce the horizontal resolution in the anisotropic LES model, the results become underdissipated when $\Delta_{h} / \Delta_{z}>4$ (not shown). This trend suggests that the ratio $\Delta_{h} / \Delta_{z}$ can also play a role in dissipation terms of anisotropic LES runs. To investigate this point further, we consider a series of additional anisotropic LES runs in the case with $\operatorname{Re}_{l}=18000$ and $N=2.1$, for which the vertical 
(a)

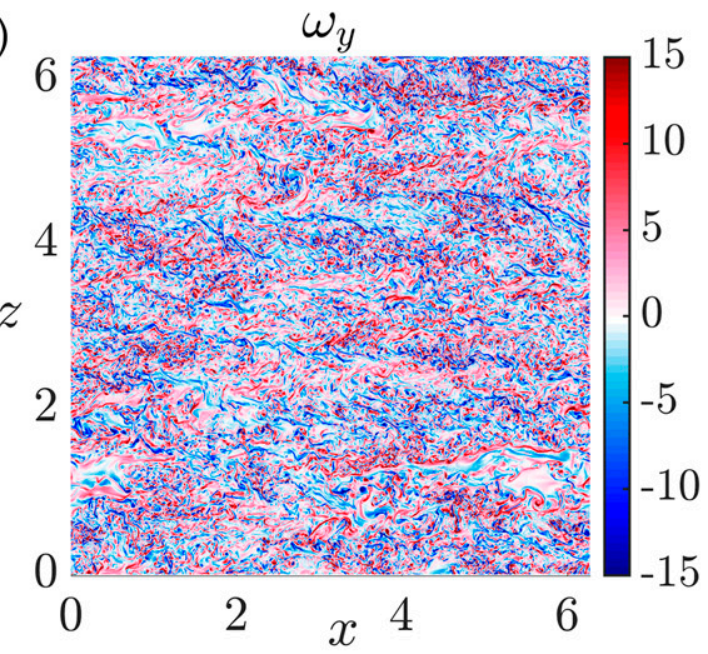

(b)

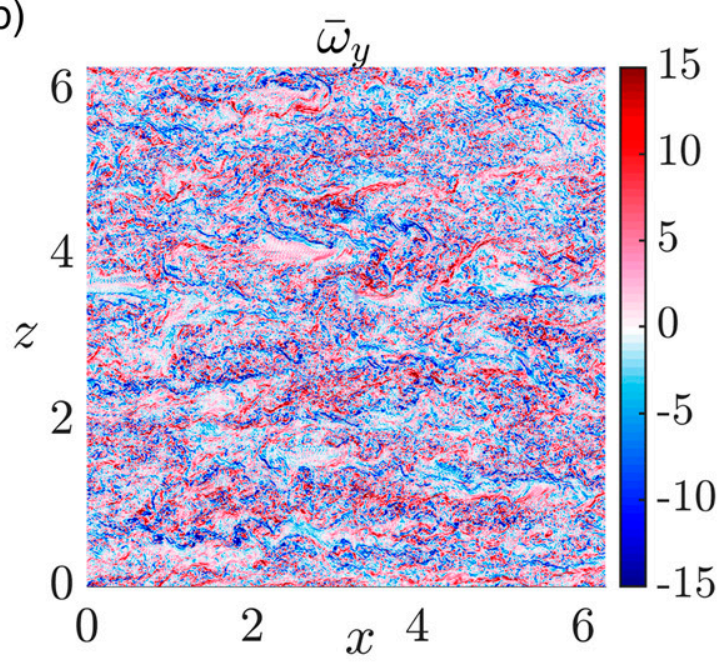

(c)

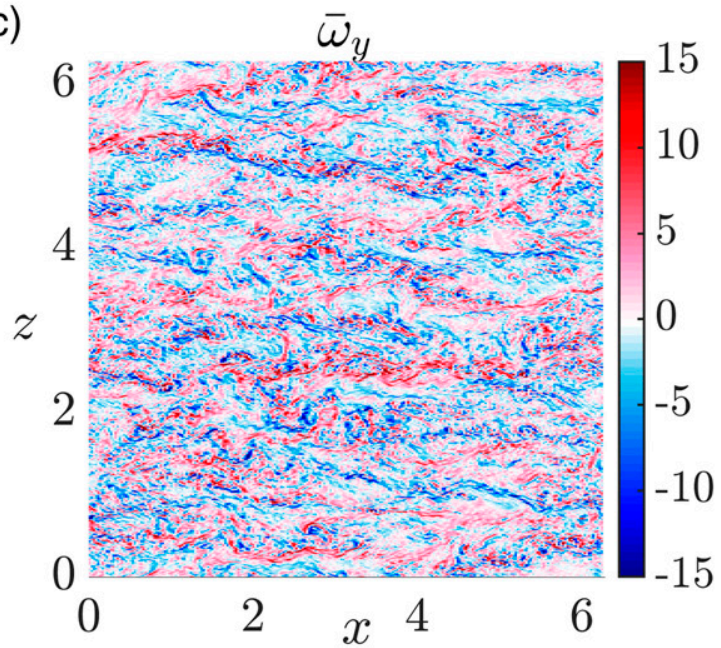

FIG. 3. Vorticity field in $y$ direction on the $x-z$ plane at $y=0.25$ and $t=15$ for the case with $\mathrm{Re}_{\ell}=18000$ and $N=2.1$ : (a) DNS, (b) high-horizontal-resolution anisotropic LES, and (c) low-horizontal-resolution anisotropic LES.

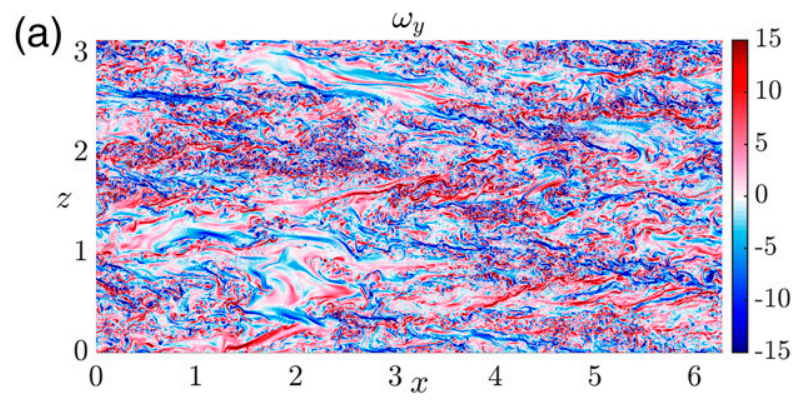

(b)

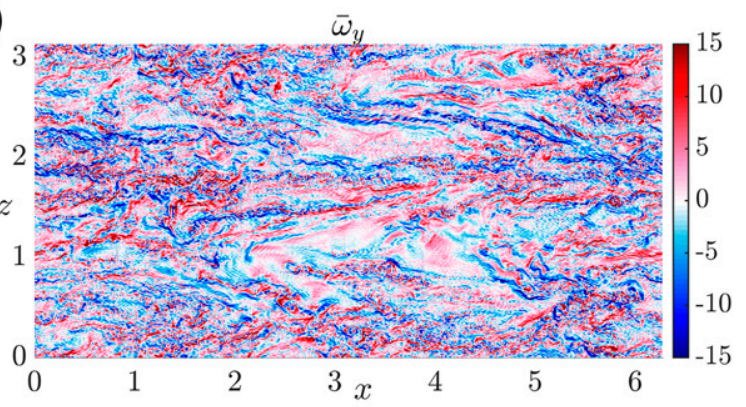

(c)

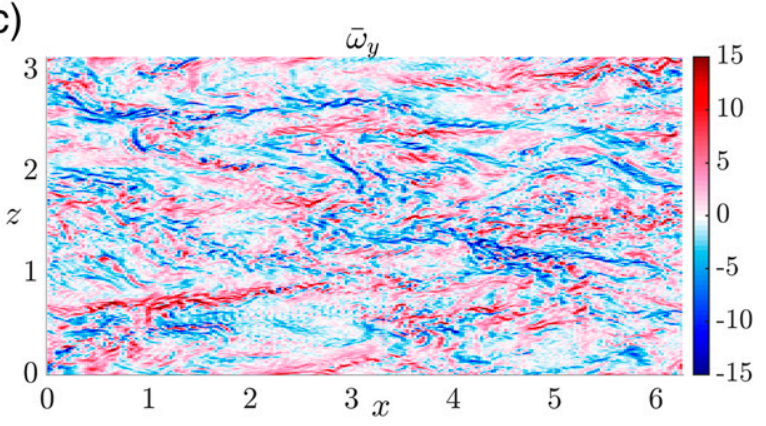

FIG. 4. Vorticity field in $y$ direction on the $x-z$ plane at $y=0.25$ and $t=15$ for the case with $\operatorname{Re}_{\ell}=22200$ and $N=4.2$ : (a) DNS, (b) high-horizontal-resolution anisotropic LES, and (c) low-horizontal-resolution anisotropic LES.

grid spacing is double the vertical grid spacing of DNS runs, with different horizontal resolution (these runs are labeled with "LAV"). Figure 6 shows the horizontal and vertical wavenumber kinetic energy spectra with $\Delta_{z}^{\mathrm{LAV}}=2 \Delta_{z}^{D}$, where cases b, $\mathrm{c}$, and d show simulations with different horizontal resolutions: that is, $\Delta_{h, b}^{\mathrm{LAV}}=1 / 2 \Delta_{h, c}^{\mathrm{LAV}}=2 / 3 \Delta_{h, d}^{\mathrm{LAV}}=\Delta_{h, b}^{\mathrm{LA}}$ (see Table 1$) . \mathrm{We}$ should mention here that the coarse horizontal resolution in cases $\mathrm{c}$ and $\mathrm{d}$ gave underdissipated results with high vertical resolution $\Delta_{z}^{D}$ (not shown), but interestingly, these cases are not underdissipated when coarser vertical grid spacing $2 \Delta_{z}^{D}$ is used (Fig. 6). Anisotropic LES with coarser vertical resolution (LAV) and DNS curves show very similar kinetic energy spectra when $k_{h}$ and $k_{v}$ are $\$ 20$. For larger wavenumbers, however, LAV runs are much more dissipative than the DNS. This behavior is expected because both horizontal and vertical grids are larger than those in DNS; for example, the curve with dash-dot green line in Fig. 6 is for a simulation with $\Delta_{z}^{\mathrm{LAV}}=2 \Delta_{z}^{D}$ and $\Delta_{h}^{\mathrm{LAV}}=8 \Delta_{h}^{D}$. In this case, we can significantly save on computational resources since this anisotropic LES run is $\approx 300$ times cheaper than 

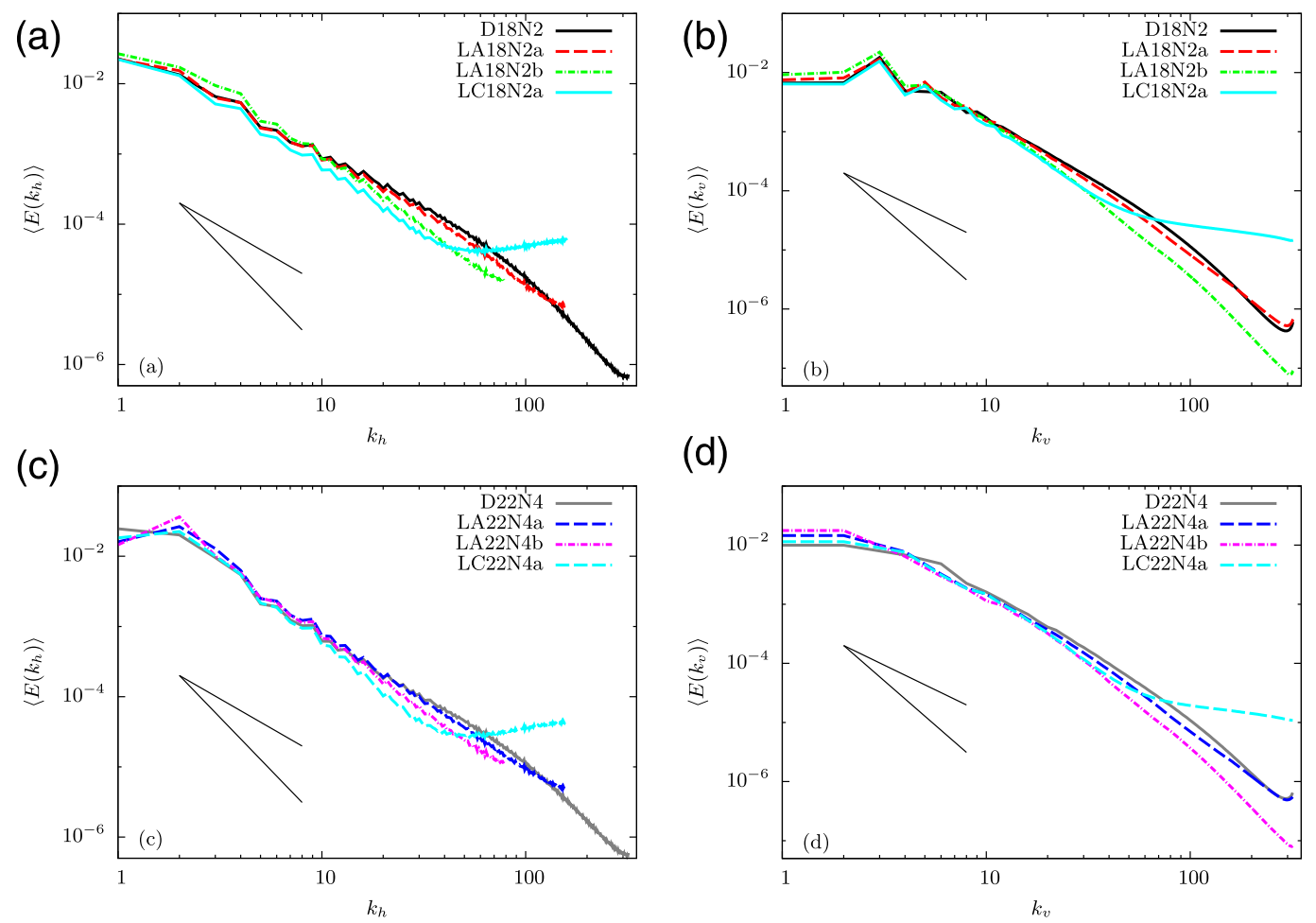

FIG. 5. The time-averaged (left) horizontal and (right) vertical wavenumber kinetic energy spectra for (top) weak and (bottom) strong stratification cases. Simulations labeled with "LC" are the same as LA, but the horizontal derivatives of vertical motions are omitted. Time averaging is performed over a window $(\Delta t=$ 4) around the maximum kinetic energy dissipation rate. The solid black line segments show $-5 / 3$ and -3 slopes.

the DNS run because of having larger grid spacing and time step for running simulations. From DNS to anisotropic LES, we can successfully reduce computational costs remarkably while the accuracy of results are still high.

Overall, we found that retaining the vertical derivatives of SGS fluxes as given by Eqs. (19)-(22) is a key in our anisotropic LES scheme to reproduce DNS results. Also, our results show that by increasing the ratio of horizontal to vertical grid spacing in the anisotropic LES model, we can help to prohibit underdissipative LES results. Nevertheless, this latter parameter setup needs to be further investigated in the realistic atmosphere and ocean (a)

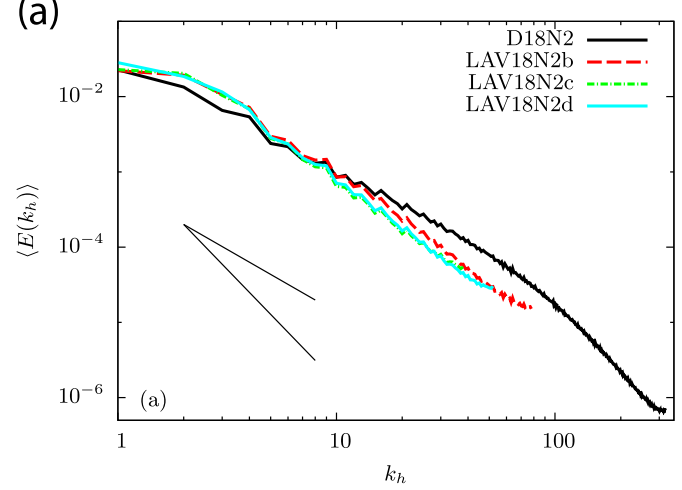

(b)

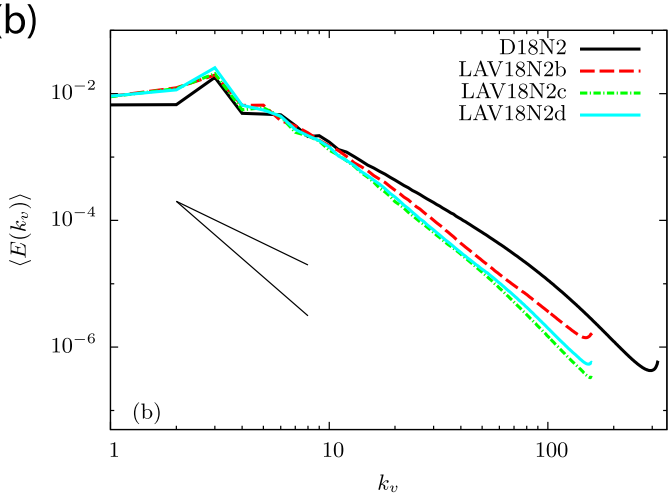

FIG. 6. The time-averaged (left) horizontal and (right) vertical wavenumber kinetic energy spectra for DNS and vertically reduced resolution anisotropic LES for the weak stratification case. Time averaging is performed over a window $(\Delta t=4)$ around the maximum kinetic energy dissipation rate. The solid black line segments show $-5 / 3$ and -3 slopes. 


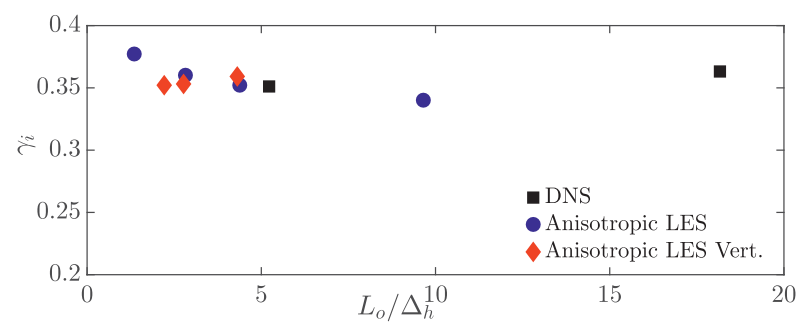

FIG. 7. Irreversible mixing efficiency $\gamma_{i}$ vs the ratio $L_{o} / \Delta_{h}$ for DNS and anisotropic LES runs. LES and LES vert. refer to those anisotropic LES runs with high and low vertical resolutions (LA and LAV), respectively.

models with our new horizontal dissipation scheme along with an appropriate vertical mixing scheme. Implementing this parameterization in atmosphere and ocean models would require geometrical adjustments for spherical coordinates (some geometrical modifications for diffusion coefficients in spherical geometry have been introduced, as in e.g., Gordon and Stern 1982; Smagorinsky 1993; Becker and Burkhardt 2007).

\section{c. Mixing efficiency}

Mixing efficiency is a key parameter in atmospheric sciences and physical oceanography, where breaking internal waves in stratified shear layers and diapycnal mixing in the upper ocean are significantly influenced by the efficiency of turbulent mixing (see e.g., Riley and Lelong 2000; Gregg et al. 2018). The irreversible mixing efficiency $\gamma_{i}$ is defined as the ratio of the molecular potential energy dissipation to the total molecular dissipation rates $\varepsilon_{p} /\left(\varepsilon+\varepsilon_{p}\right)$ (Winters and D'Asaro 1996; Caulfield and Peltier 2000). This quantity has been extended to be used in LES with SGS eddy dissipation rates (Khani 2018). The SGS mixing efficiency $\gamma_{i}$ depends on the turbulent Prandtl number $\operatorname{Pr}_{t}$ as follows:

$$
\gamma_{i} \sim \frac{1}{1+2 \operatorname{Pr}_{t}},
$$

implying $\gamma_{i} \approx 1 / 3$ in stratified turbulence with $\operatorname{Pr}_{t}=1$ (Khani 2018).

Figure 7 shows the irreversible mixing efficiency $\gamma_{i}$ versus the resolution of the Ozmidov scale $L_{o}$ in the horizontal direction, for DNS and anisotropic LES approaches. As expected, the ratio $L_{o} / \Delta_{h}$ is larger in DNS compared to that in LES. Nevertheless, values of $\gamma_{i}$ in LES overlap well with those from DNS and are in line with the theoretical estimate of $1 / 3$ for LES of stratified turbulence (Fig. 7). This agreement is due to the resolution of the Ozmidov scale $L_{o}$ in LES runs (see Khani (2018) for more information). Noteworthy, unlike in the simulations in Khani (2018), where only large-scale vortical modes were initially excited, here we excite large horizontal and vertical motions, which results in more efficient energy exchange between KE and PE through the buoyancy fluxes. In this case, $\gamma_{i}$ is slightly larger than $1 / 3$ for both DNS and LES runs (Fig. 7).

\section{Conclusions}

A new anisotropic SGS model in LES of stratified turbulence is introduced. The new scheme uses coarse grid spacing in the horizontal direction, and also retains the vertical derivatives of horizontal motions in the eddy dissipation terms, which are omitted in the classic LES approach for horizontal dissipation. Therefore, our new model maintains anisotropy in the resolution, and the connection between the horizontal and vertical motions in the eddy dissipation. The new anisotropic SGS parameterization is tested in LES of decaying stratified turbulence, and the results are compared with those from DNS: the time series of total energy and kinetic energy dissipation rate, vorticity field, horizontal and vertical wavenumber spectra, and mixing efficiency are fairly well reproduced in the new LES scheme similar to those in DNS, while the computational cost is largely decreased in LES.

It has been shown that if we neglect the vertical derivatives of SGS motions in our eddy dissipation terms, our results will be underdissipated at small scales. We think a similar story should exist in atmosphere and ocean models (e.g., Griffies and Hallberg 2000; Griffies et al. 2004; Skamarock et al. 2008), where the vertical derivatives of SGS fluxes are neglected by horizontal mixing schemes. As a result, we hypothesize that the horizontal eddy dissipation parameters may sometimes be artificially increased in atmosphere and ocean models to ensure model convergence since the zonal and meridional SGS eddy fluxes do not include fluxes from vertical motions. This unrealistically enhanced horizontal eddy dissipation can affect the results of atmosphere and ocean models, and may be compensated by adding an energizing term in the form of a stochastic or negative Laplacian backscatter (as in Mana and Zanna 2014; Jansen and Held 2014) to the equations of motion, in order to improve the performance of these models. Our work suggests that if we keep the neglected terms in the horizontal eddy dissipation scheme, the model performance may be improved without adding any additional energizing terms to the zonal and meridional momentum equations. Nevertheless, this suggestion has to be tested in large-scale atmosphere and ocean models since the flow regime in such models, even at the grid scale, is affected by rotating, unlike the stratified turbulence considered here. In addition, as model resolutions continue to increase, gridscale motions in such models will become closer to the stratified turbulence regime, and our findings will become increasingly relevant.

In atmosphere and ocean models, different types of SGS eddy viscosity and diffusivity parameterizations can be used. In addition to the Smagorinsky model, a common SGS model in atmosphere and ocean simulations is the turbulent kinetic energy (TKE) model, where the term $\bar{S}$ in Eqs. (23)-(26) is replaced by $K_{\mathrm{tke}}=c_{k} \mathscr{C}_{m} \bar{e}^{1 / 2}$. Here, $c_{k}$ is a constant coefficient, $\mathscr{L}_{m}$ is a mixing length that is usually proportional to the grid spacing, and $\bar{e}$ is the SGS turbulence kinetic energy that is computed by solving a transport equation for the TKE budget (see e.g., Sommeria 1976; Deardorff 1980; Schumann 1991; 
Kaltenbach et al. 1994). Our anisotropic horizontal-filtering framework can be easily adapted to such models. Furthermore, our approach can also be used in more complicated atmosphere and ocean models where, for example, water vapor or other scalars are considered. For these cases, we would need to perform scale analysis for the SGS fluxes, which are proportional to the gradient of corresponding quantities, and include the vertical derivatives of quantities in the horizontally filtered transport equations. Meanwhile, we should evaluate the performance of our anisotropic SGS parameterization in atmosphere and ocean simulations, which have much lower vertical resolution than what we consider here, with a hierarchy of vertical subgrid schemes. We will consider simulations with and without current vertical SGS parameterizations in atmosphere and ocean models. Also, depending on the ratio of $\Delta_{h} / \Delta_{z}$, we can develop a new SGS scheme based on a vertical-filtering approach. For future work, we plan to test the performance of our new anisotropic SGS parameterization in atmosphere and ocean models considering these modifications and complexities.
Acknowledgments. This paper has benefited from comments by Steve Griffies, Almut Gassmann, and three anonymous reviewers. This research was enabled in part by support provided by the GPC supercomputer at the SciNet HPC Consortium, Shared Hierarchical Academic Research Computing Network (SHARCNET), and Compute Canada (www.computecanada.ca). S.K. gratefully acknowledges the financial support provided by National Science Foundation through Awards 1536360 and 1536314. M.L.W. gratefully acknowledges support from the Natural Sciences and Engineering Research Council of Canada (Grant RGPIN386456-2015). Model data used in this study are available upon request to the corresponding author.

\section{APPENDIX A}

\section{A Nonlinear Approximation for the SGS Stress $\tau_{i j}$}

Using the Taylor series expansion of the velocity field $\mathbf{u}$, the nonlinear tensor $u_{i}(\mathbf{x}+\mathbf{r}) u_{j}(\mathbf{x}+\mathbf{r})$ can also be expanded, keeping up to cubic terms in $\mathbf{r}$, as ${ }^{\mathrm{A} 1}$

$$
\begin{aligned}
u_{i}(\mathbf{x}+\mathbf{r}) u_{j}(\mathbf{x}+\mathbf{r})= & u_{i}(\mathbf{x}) u_{j}(\mathbf{x})+\frac{\partial\left[u_{i}(\mathbf{x}) u_{j}(\mathbf{x})\right]}{\partial x_{k}} r_{k}+\frac{\partial u_{i}(\mathbf{x})}{\partial x_{k}} \frac{\partial u_{j}(\mathbf{x})}{\partial x_{l}} r_{k} r_{l}+\frac{1}{2} u_{j}(\mathbf{x}) \frac{\partial^{2} u_{i}(\mathbf{x})}{\partial x_{k} \partial x_{l}} r_{k} r_{l}+\frac{1}{2} u_{i}(\mathbf{x}) \frac{\partial^{2} u_{j}(\mathbf{x})}{\partial x_{k} \partial x_{l}} r_{k} r_{l} \\
& +\frac{1}{2} \frac{\partial u_{i}(\mathbf{x})}{\partial x_{m}} \frac{\partial^{2} u_{j}(\mathbf{x})}{\partial x_{k} \partial x_{l}} r_{m} r_{k} r_{l}+\frac{1}{2} \frac{\partial u_{j}(\mathbf{x})}{\partial x_{m}} \frac{\partial^{2} u_{i}(\mathbf{x})}{\partial x_{k} \partial x_{l}} r_{m} r_{k} r_{l}+\frac{1}{6} u_{i}(\mathbf{x}) \frac{\partial^{3} u_{j}(\mathbf{x})}{\partial x_{m} \partial x_{k} \partial x_{l}} r_{m} r_{k} r_{l}+\frac{1}{6} u_{j}(\mathbf{x}) \frac{\partial^{3} u_{i}(\mathbf{x})}{\partial x_{m} \partial x_{k} \partial x_{l}} r_{m} r_{k} r_{l}+\ldots
\end{aligned}
$$

We can apply the filter function $G(\mathbf{r})$ to Eqs. (10) and (A1), respectively, and integrate over the domain $D$ in order to find the filtered variables. For velocities $u_{i}(\mathbf{x})$ and $u_{j}(\mathbf{x})$, keeping up to the cubic terms in the Taylor series, we have

$$
\begin{aligned}
\bar{u}_{i}(\mathbf{x}) \approx & u_{i}(\mathbf{x})+\frac{\partial u_{i}(\mathbf{x})}{\partial x_{k}} \int_{D} r_{k} G(\mathbf{r}) d \mathbf{r}+\frac{1}{2} \frac{\partial^{2} u_{i}(\mathbf{x})}{\partial x_{k} \partial x_{l}} \int_{D} r_{k} r_{l} G(\mathbf{r}) d \mathbf{r} \\
& +\frac{1}{6} \frac{\partial^{3} u_{i}(\mathbf{x})}{\partial x_{m} \partial x_{k} \partial x_{l}} \int_{D} r_{k} r_{l} r_{m} G(\mathbf{r}) d \mathbf{r}
\end{aligned}
$$

$$
\begin{aligned}
\bar{u}_{j}(\mathbf{x}) \approx & u_{j}(\mathbf{x})+\frac{\partial u_{j}(\mathbf{x})}{\partial x_{k}} \int_{D} r_{k} G(\mathbf{r}) d \mathbf{r}+\frac{1}{2} \frac{\partial^{2} u_{j}(\mathbf{x})}{\partial x_{k} \partial x_{l}} \int_{D} r_{k} r_{l} G(\mathbf{r}) d \mathbf{r} \\
& +\frac{1}{6} \frac{\partial^{3} u_{j}(\mathbf{x})}{\partial x_{m} \partial x_{k} \partial x_{l}} \int_{D} r_{k} r_{l} r_{m} G(\mathbf{r}) d \mathbf{r}
\end{aligned}
$$

Similarly for $u_{i}(\mathbf{x}) u_{j}(\mathbf{x})$, keeping up to cubic terms, we have

$$
\begin{aligned}
\overline{u_{i}(\mathbf{x}) u_{j}(\mathbf{x})} \approx & u_{i}(\mathbf{x}) u_{j}(\mathbf{x})+\frac{\partial\left[u_{i}(\mathbf{x}) u_{j}(\mathbf{x})\right]}{\partial x_{k}} \int_{D} r_{k} G(\mathbf{r}) d \mathbf{r}+\frac{\partial u_{i}(\mathbf{x})}{\partial x_{l}} \frac{\partial u_{j}(\mathbf{x})}{\partial x_{k}} \int_{D} r_{k} r_{l} G(\mathbf{r}) d \mathbf{r}+\frac{1}{2} u_{i}(\mathbf{x}) \frac{\partial^{2} u_{j}(\mathbf{x})}{\partial x_{k} \partial x_{l}} \int_{D} r_{k} r_{l} G(\mathbf{r}) d \mathbf{r} \\
& +\frac{1}{2} u_{j}(\mathbf{x}) \frac{\partial^{2} u_{i}(\mathbf{x})}{\partial x_{k} \partial x_{l}} \int_{D} r_{k} r_{l} G(\mathbf{r}) d \mathbf{r}+\frac{1}{2} \frac{\partial u_{i}(\mathbf{x})}{\partial x_{m}} \frac{\partial^{2} u_{j}(\mathbf{x})}{\partial x_{k} \partial x_{l}} \int_{D} r_{k} r_{l} r_{m} G(\mathbf{r}) d \mathbf{r}+\frac{1}{2} \frac{\partial u_{j}(\mathbf{x})}{\partial x_{m}} \frac{\partial^{2} u_{i}(\mathbf{x})}{\partial x_{k} \partial x_{l}} \int_{D} r_{k} r_{l} r_{m} G(\mathbf{r}) d \mathbf{r} \\
& +\frac{1}{6} u_{i}(\mathbf{x}) \frac{\partial^{3} u_{j}(\mathbf{x})}{\partial x_{m} \partial x_{k} \partial x_{l}} \int_{D} r_{k} r_{l} r_{m} G(\mathbf{r}) d \mathbf{r}+\frac{1}{6} u_{j}(\mathbf{x}) \frac{\partial^{3} u_{i}(\mathbf{x})}{\partial x_{m} \partial x_{k} \partial x_{l}} \int_{D} r_{k} r_{l} r_{m} G(\mathbf{r}) d \mathbf{r} .
\end{aligned}
$$

Using Eqs. (A2) and (A3), we can also approximate the nonlinear filtered product $\bar{u}_{i}(\mathbf{x}) \bar{u}_{j}(\mathbf{x})$, keeping up to cubic terms, as follows:

\footnotetext{
${ }^{\text {A1 }}$ For simplicity, partial derivatives with respect to the variable $\left.r_{k}\right|_{r_{k}=0}$ are written as partial derivatives with respect to $x_{k}$ because term $\mathbf{x}+\mathbf{r}$ reduces to $\mathbf{x}$ when $\mathbf{r}=0$ (as in e.g., Pope 2000).
} 


$$
\begin{aligned}
& \bar{u}_{i}(\mathbf{x}) \bar{u}_{j}(\mathbf{x}) \approx u_{i}(\mathbf{x}) u_{j}(\mathbf{x})+\frac{\partial\left[u_{i}(\mathbf{x}) u_{j}(\mathbf{x})\right]}{\partial x_{k}} \int_{D} r_{k} G(\mathbf{r}) d \mathbf{r}+\left[\frac{\partial u_{i}(\mathbf{x})}{\partial x_{l}} \int_{D} r_{l} G(\mathbf{r}) d \mathbf{r}\right]\left[\frac{\partial u_{j}(\mathbf{x})}{\partial x_{k}} \int_{D} r_{k} G(\mathbf{r}) d \mathbf{r}\right]+\frac{1}{2} u_{i}(\mathbf{x}) \frac{\partial^{2} u_{j}(\mathbf{x})}{\partial x_{k} \partial x_{l}} \int_{D} r_{k} r_{l} G(\mathbf{r}) d \mathbf{r} \\
& +\frac{1}{2} u_{j}(\mathbf{x}) \frac{\partial^{2} u_{i}(\mathbf{x})}{\partial x_{k} \partial x_{l}} \int_{D} r_{k} r_{l} G(\mathbf{r}) d \mathbf{r}+\frac{1}{2}\left[\frac{\partial u_{i}(\mathbf{x})}{\partial x_{m}} \int_{D} r_{m} G(\mathbf{r}) d \mathbf{r}\right]\left[\frac{\partial^{2} u_{j}(\mathbf{x})}{\partial x_{k} \partial x_{l}} \int_{D} r_{k} r_{l} G(\mathbf{r}) d \mathbf{r}\right]+\frac{1}{2}\left[\frac{\partial u_{j}(\mathbf{x})}{\partial x_{m}} \int_{D} r_{m} G(\mathbf{r}) d \mathbf{r}\right] \\
& \times\left[\frac{\partial^{2} u_{i}(\mathbf{x})}{\partial x_{k} \partial x_{l}} \int_{D} r_{k} r_{l} G(\mathbf{r}) d \mathbf{r}\right]+\frac{1}{6} u_{i}(\mathbf{x}) \frac{\partial^{3} u_{j}(\mathbf{x})}{\partial x_{m} \partial x_{k} \partial x_{l}} \int_{D} r_{k} r_{l} r_{m} G(\mathbf{r}) d \mathbf{r}+\frac{1}{6} u_{j}(\mathbf{x}) \frac{\partial^{3} u_{i}(\mathbf{x})}{\partial x_{m} \partial x_{k} \partial x_{l}} \int_{D} r_{k} r_{l} r_{m} G(\mathbf{r}) d \mathbf{r} .
\end{aligned}
$$

Subtracting Eq. (A5) from Eq. (A4) results in a mathematical formulation for the approximate SGS stress $\tau_{i j}(\mathbf{x})=$ $\overline{u_{i}(\mathbf{x}) u_{j}(\mathbf{x})}-\bar{u}_{i}(\mathbf{x}) \bar{u}_{j}(\mathbf{x})$, written as

$$
\tau_{i j}(\mathbf{x})=\frac{\partial u_{i}(\mathbf{x})}{\partial x_{l}} \frac{\partial u_{j}(\mathbf{x})}{\partial x_{k}} \int_{D} r_{k} r_{l} G(\mathbf{r}) d \mathbf{r}+\mathscr{O}\left(\Delta^{4}\right)
$$

where we have assumed that the odd moments of the filter function $G(\mathbf{r})$ are zero, and $\mathscr{O}\left(\Delta^{4}\right)$ shows the order of leading error in this approximation, since the fourth-order moments of $G(\mathbf{r})$ are proportional to $\Delta^{4}$.

\section{APPENDIX B}

\section{A Nonlinear Approximation for the SGS Flux $\boldsymbol{h}_{\boldsymbol{j}}$}

We can expand the potential temperature field $\theta$ using the Taylor series at a given point $\mathbf{x}$ in $\mathbf{r}$, which is of the order filter width $\Delta$ (again we keep up to cubic terms):

$$
\begin{aligned}
\theta(\mathbf{x}+\mathbf{r})= & \theta(\mathbf{x})+\frac{\partial \theta(\mathbf{x})}{\partial x_{k}} r_{k}+\frac{1}{2} \frac{\partial^{2} \theta(\mathbf{x})}{\partial x_{k} \partial x_{l}} r_{k} r_{l} \\
& +\frac{1}{6} \frac{\partial^{3} \theta(\mathbf{x})}{\partial x_{m} \partial x_{k} \partial x_{l}} r_{m} r_{k} r_{l}+\cdots .
\end{aligned}
$$

If we employ the filter function $G(\mathbf{r})$ to the Eq. (B1), and keep up to cubic terms, we obtain

$$
\begin{aligned}
\bar{\theta}(\mathbf{x}) \approx & \theta(\mathbf{x})+\frac{\partial \theta(\mathbf{x})}{\partial x_{k}} \int_{D} r_{k} G(\mathbf{r}) d \mathbf{r}+\frac{1}{2} \frac{\partial^{2} \theta(\mathbf{x})}{\partial x_{k} \partial x_{l}} \int_{D} r_{k} r_{l} G(\mathbf{r}) d \mathbf{r} \\
& +\frac{1}{6} \frac{\partial^{3} \theta(\mathbf{x})}{\partial x_{m} \partial x_{k} \partial x_{l}} \int_{D} r_{m} r_{k} r_{l} G(\mathbf{r}) d \mathbf{r} .
\end{aligned}
$$

Similarly, we can expand the SGS potential temperature flux $\overline{\theta(\mathbf{x}) u_{j}(\mathbf{x})}$ and resolved potential temperature flux $\bar{\theta}(\mathbf{x}) \bar{u}_{j}(\mathbf{x})$, keeping up to cubic terms, in the following forms:

$$
\begin{aligned}
\overline{\theta(\mathbf{x}) u_{j}(\mathbf{x}) \approx} & \theta(\mathbf{x}) u_{j}(\mathbf{x})+\frac{\partial\left[\theta(\mathbf{x}) u_{j}(\mathbf{x})\right]}{\partial x_{k}} \int_{D} r_{k} G(\mathbf{r}) d \mathbf{r}+\frac{\partial \theta(\mathbf{x})}{\partial x_{l}} \frac{\partial u_{j}(\mathbf{x})}{\partial x_{k}} \int_{D} r_{k} r_{l} G(\mathbf{r}) d \mathbf{r}+\frac{1}{2} \theta(\mathbf{x}) \frac{\partial^{2} u_{j}(\mathbf{x})}{\partial x_{k} \partial x_{l}} \int_{D} r_{k} r_{l} G(\mathbf{r}) d \mathbf{r} \\
& +\frac{1}{2} u_{j}(\mathbf{x}) \frac{\partial^{2} \theta(\mathbf{x})}{\partial x_{k} \partial x_{l}} \int_{D} r_{k} r_{l} G(\mathbf{r}) d \mathbf{r}+\frac{1}{2} \frac{\partial \theta(\mathbf{x})}{\partial x_{m}} \frac{\partial^{2} u_{j}(\mathbf{x})}{\partial x_{k} \partial x_{l}} \int_{D} r_{k} r_{l} r_{m} G(\mathbf{r}) d \mathbf{r}+\frac{1}{2} \frac{\partial u_{j}(\mathbf{x})}{\partial x_{m}} \frac{\partial^{2} \theta(\mathbf{x})}{\partial x_{k} \partial x_{l}} \int_{D} r_{k} r_{l} r_{m} G(\mathbf{r}) d \mathbf{r} \\
& +\frac{1}{6} \theta(\mathbf{x}) \frac{\partial^{3} u_{j}(\mathbf{x})}{\partial x_{m} \partial x_{k} \partial x_{l}} \int_{D} r_{k} r_{l} r_{m} G(\mathbf{r}) d \mathbf{r}+\frac{1}{6} u_{j}(\mathbf{x}) \frac{\partial^{3} \theta(\mathbf{x})}{\partial x_{m} \partial x_{k} \partial x_{l}} \int_{D} r_{k} r_{l} r_{m} G(\mathbf{r}) d \mathbf{r},
\end{aligned}
$$

and

$$
\begin{aligned}
& \bar{\theta}(\mathbf{x}) \bar{u}_{j}(\mathbf{x}) \approx \theta(\mathbf{x}) u_{j}(\mathbf{x})+\frac{\partial\left[\theta(\mathbf{x}) u_{j}(\mathbf{x})\right]}{\partial x_{k}} \int_{D} r_{k} G(\mathbf{r}) d \mathbf{r}+\left[\frac{\partial \theta(\mathbf{x})}{\partial x_{l}} \int_{D} r_{l} G(\mathbf{r}) d \mathbf{r}\right]\left[\frac{\partial u_{j}(\mathbf{x})}{\partial x_{k}} \int_{D} r_{k} G(\mathbf{r}) d \mathbf{r}\right]+\frac{1}{2} \theta(\mathbf{x}) \frac{\partial^{2} u_{j}(\mathbf{x})}{\partial x_{k} \partial x_{l}} \int_{D} r_{k} r_{l} G(\mathbf{r}) d \mathbf{r} \\
& +\frac{1}{2} u_{j}(\mathbf{x}) \frac{\partial^{2} \theta(\mathbf{x})}{\partial x_{k} \partial x_{l}} \int_{D} r_{k} r_{l} G(\mathbf{r}) d \mathbf{r}+\frac{1}{2}\left[\frac{\partial \theta(\mathbf{x})}{\partial x_{m}} \int_{D} r_{m} G(\mathbf{r}) d \mathbf{r}\right]\left[\frac{\partial^{2} u_{j}(\mathbf{x})}{\partial x_{k} \partial x_{l}} \int_{D} r_{k} r_{l} G(\mathbf{r}) d \mathbf{r}\right] \\
& +\frac{1}{2}\left[\frac{\partial u_{j}(\mathbf{x})}{\partial x_{m}} \int_{D} r_{m} G(\mathbf{r}) d \mathbf{r}\right]\left[\frac{\partial^{2} \theta(\mathbf{x})}{\partial x_{k} \partial x_{l}} \int_{D} r_{k} r_{l} G(\mathbf{r}) d \mathbf{r}\right]+\frac{1}{6} \theta(\mathbf{x}) \frac{\partial^{3} u_{j}(\mathbf{x})}{\partial x_{m} \partial x_{k} \partial x_{l}} \int_{D} r_{k} r_{l} r_{m} G(\mathbf{r}) d \mathbf{r}+\frac{1}{6} u_{j}(\mathbf{x}) \frac{\partial^{3} \theta(\mathbf{x})}{\partial x_{m} \partial x_{k} \partial x_{l}} \int_{D} r_{k} r_{l} r_{m} G(\mathbf{r}) d \mathbf{r} .
\end{aligned}
$$

Therefore, the SGS potential temperature flux term $h_{j}(\mathbf{x})=$ $\overline{\theta(\mathbf{x}) u_{j}(\mathbf{x})}-\bar{\theta}(\mathbf{x}) \bar{u}_{j}(\mathbf{x})$ is

$$
h_{j}(\mathbf{x})=\frac{\partial \theta(\mathbf{x})}{\partial x_{l}} \frac{\partial u_{j}(\mathbf{x})}{\partial x_{k}} \int_{D} r_{k} r_{l} G(\mathbf{r}) d \mathbf{r}+\mathscr{O}\left(\Delta^{4}\right),
$$

where we have again assumed that the odd moments of the filter function $G(\mathbf{r})$ are zero.

\section{REFERENCES}

Augier, P., and E. Lindborg, 2013: A new formulation of the spectral energy budget of the atmosphere, with application to 
two high-resolution general circulation models. J. Atmos. Sci., 70, 2293-2308, https://doi.org/10.1175/JAS-D-12-0281.1.

Bartello, P., and M. Tobias, 2013: Sensitivity of stratified turbulence to the buoyancy Reynolds number. J. Fluid Mech., 725, 1-22, https://doi.org/10.1017/jfm.2013.170.

Becker, E., and U. Burkhardt, 2007: Nonlinear horizontal diffusion for GCMs. Mon. Wea. Rev., 135, 1439-1454, https://doi.org/ 10.1175/MWR3348.1.

Brethouwer, G., P. Billant, E. Lindborg, and J.-M. Chomaz, 2007: Scaling analysis and simulation of strongly stratified turbulent flows. J. Fluid Mech., 585, 343-368, https://doi.org/10.1017/ S0022112007006854.

Brune, S., and E. Becker, 2013: Indications of stratified turbulence in a mechanistic GCM. J. Atmos. Sci., 70, 231-247, https:// doi.org/10.1175/JAS-D-12-025.1.

Caulfield, C. P., and W. R. Peltier, 2000: The anatomy of the mixing transition in homogeneous and stratified free shear layers. J. Fluid Mech., 413, 1-47, https://doi.org/10.1017/ S0022112000008284.

Deardorff, J. W., 1980: Stratocumulus-capped mixed layers derived from a three-dimensional model. Bound.-Layer Meteor., 18, 495-527, https://doi.org/10.1007/BF00119502.

Durran, D. R., 2010: Numerical Methods for Fluid Dynamics with Application to Geophysics. Springer, $516 \mathrm{pp}$.

Gordon, C. T., and W. F. Stern, 1982: A description of the GFDL global spectral model. Mon. Wea. Rev., 110, 625-644, https://doi.org/10.1175/1520-0493(1982)110<0625:ADOTGG> 2.0.CO;2.

Gregg, M. C., E. A. D’Asaro, J. J. Riley, and E. Kunze, 2018: Mixing efficiency in the ocean. Annu. Rev. Mar. Sci., 10, 443-473, https://doi.org/10.1146/annurev-marine-121916-063643.

Griffies, S. M., and R. Hallberg, 2000: Biharmonic friction with a Smagorinsky-like viscosity for use in large-scale eddypermitting ocean models. Mon. Wea. Rev., 128, 2935-2946, https://doi.org/10.1175/1520-0493(2000)128<2935:BFWASL> 2.0.CO;2.

—, M. J. Harrison, R. C. Pacanowski, and A. Rosati, 2004: A technical guide to MOM4. GFDL Ocean Group Tech. Rep. 5, NOAA/GFDL, 342 pp., https://www.gfdl.noaa.gov/bibliography/ related_files/smg0301.pdf.

Jansen, M. F., and I. M. Held, 2014: Parameterizing subgrid-scale eddy effects using energetically consistent backscatter. Ocean Modell., 80, 36-48, https://doi.org/10.1016/j.ocemod.2014.06.002.

Kaltenbach, H.-J., T. Gerz, and U. Schumann, 1994: Large-eddy simulation of homogeneous turbulence and diffusion in stably stratified shear flow. J. Fluid Mech., 280, 1-40, https://doi.org/ 10.1017/S0022112094002831.

Kang, H. S., S. Chester, and C. Meneveau, 2003: Decaying turbulence in an active-grid-generated flow and comparisons with large-eddy simulation. J. Fluid Mech., 480, 129-160, https:// doi.org/10.1017/S0022112002003579.

Khani, S., 2018: Mixing efficiency in large-eddy simulations of stratified turbulence. J. Fluid Mech., 849, 373-394, https:// doi.org/10.1017/jfm.2018.417.

—_, and M. L. Waite, 2013: Effective eddy viscosity in stratified turbulence. J. Turbul., 14, 49-70, https://doi.org/10.1080/ 14685248.2013.837913.

— , and — 2014: Buoyancy scale effects in large-eddy simulations of stratified turbulence. J. Fluid Mech., 754, 75-97, https://doi.org/10.1017/jfm.2014.381.

— lence: The dynamic Smagorinsky model. J. Fluid Mech., 773, 327-344, https://doi.org/10.1017/jfm.2015.249.
—_, and ——, 2016: Backscatter in stratified turbulence. Eur. J. Mech. B/Fluids, 60, 1-12, https://doi.org/10.1016/ j.euromechflu.2016.06.012.

_ subgrid-scale models in stratified turbulence using direct numerical simulations. Eur. J. Mech. B/Fluids, 65, 168-178, https://doi.org/10.1016/j.euromechflu.2017.03.009.

—_, and —_, 2017b: A modulated-gradient parametrization for the large-eddy simulation of the atmospheric boundary layer using the Weather Research and Forecasting model. Bound.-Layer Meteor., 165, 385-404, https://doi.org/10.1007/ s10546-017-0287-5.

Lindborg, E., 2006: The energy cascade in a strongly stratified fluid. J. Fluid Mech., 550, 207-242, https://doi.org/10.1017/ S0022112005008128.

Mana, P. P., and L. Zanna, 2014: Toward a stochastic parameterization of ocean mesoscale eddies. Ocean Modell., 79, 1-20, https://doi.org/10.1016/j.ocemod.2014.04.002.

Meneveau, C., and J. Katz, 2000: Scale-invariance and turbulence models for large-eddy simulation. Annu. Rev. Fluid Mech., 32, 1-32, https://doi.org/10.1146/annurev.fluid.32.1.1.

Moin, P., and K. Mahesh, 1998: Direct numerical simulation: A tool in turbulence research. Rev. Fluid Mech., 30, 539-578, https:// doi.org/10.1146/annurev.fluid.30.1.539.

Orszag, S. A., 1971: On the elimination of aliasing in finite-difference schemes by filtering high-wavenumber components. J. Atmos. Sci., 28, 1074, https://doi.org/10.1175/1520-0469(1971)028<1074: OTEOAI $>2.0 . \mathrm{CO} ; 2$.

Paoli, R., O. Thouron, J. Escobar, J. Picot, and D. Cariolle, 2014: High-resolution large-eddy simulations of stably stratified flows: Application to subkilometer-scale turbulence in the upper troposphere-lower stratosphere. Atmos. Chem. Phys., 14, 5037-5055, https://doi.org/10.5194/acp-145037-2014.

Pope, S. B., 2000: Turbulent Flows. Cambridge University Press, $771 \mathrm{pp}$.

Riley, J. J., and M.-P. Lelong, 2000: Fluid motions in the presence of strong stable stratification. Annu. Rev. Fluid Mech., 32, 613-657, https://doi.org/10.1146/annurev.fluid.32.1.613.

—_, and E. Lindborg, 2008: Stratified turbulence: A possible interpretation of some geophysical turbulence measurements. J. Atmos. Sci., 65, 2416-2424, https://doi.org/10.1175/ 2007JAS2455.1.

Schaefer-Rolffs, U., and E. Becker, 2018: Scale-invariant formulation of momentum diffusion for high-resolution atmospheric circulation models. Mon. Wea. Rev., 146, 1045-1062, https:// doi.org/10.1175/MWR-D-17-0216.1.

Schumann, U., 1991: Subgrid length-scale for large-eddy simulation of stratified turbulence. Theor. Comput. Fluid Dyn., 2, 279-290, https://doi.org/10.1007/BF00271468.

Siegel, D. A., and J. A. Domaradzki, 1994: Large-eddy simulation of stably stratified turbulence. J. Phys. Oceanogr., 24, 2353-2386, https://doi.org/10.1175/1520-0485(1994)024<2353: LESODS $>2.0$.CO;2.

Skamarock, W. C., and Coauthors, 2008: A description of the Advanced Research WRF version 3. NCAR Tech. Note NCAR/TN-475+STR, 113 pp., https://doi.org/10.5065/ D68S4MVH.

— C. Snyder, J. B. Klemp, and S.-H. Park, 2019: Vertical resolution requirements in atmospheric simulation. Mon. Wea. Rev., 147, 2641-2656, https://doi.org/10.1175/MWR-D-19-0043.1.

Smagorinsky, J., 1993: Some historical remarks on the use of nonlinear viscosities. Large Eddy Simulation of Complex 
Engineering and Geophysical Flows, B. Galperin and St. A. Orszag, Eds., Cambridge University Press, 3-36.

Sommeria, G., 1976: Three-dimensional simulation of turbulence processes in an undisturbed trade wind boundary layer. J. Atmos. Sci., 33, 216-241, https://doi.org/10.1175/ 1520-0469(1976)033<0216:TDSOTP $>2.0$. CO;2.

Waite, M. L., 2011: Stratified turbulence at the buoyancy scale. Phys. Fluids, 23, 066602, https://doi.org/10.1063/ 1.3599699 .
- 2016: Dependence of model energy spectra on vertical resolution. Mon. Wea. Rev., 144, 1407-1421, https://doi.org/ 10.1175/MWR-D-15-0316.1.

_ and P. Bartello, 2004: Stratified turbulence dominated by vortical motion. J. Fluid Mech., 517, 281-308, https://doi.org/ 10.1017/S0022112004000977.

Winters, K. B., and E. A. D'Asaro, 1996: Diascalar flux and the rate of fluid mixing. J. Fluid Mech., 317, 179-193, https://doi.org/ 10.1017/S0022112096000717. 